\title{
Mild cognitive impairment: a cross-national comparison
}

\author{
E Arnáiz, O Almkvist, R J lvnik, E G Tangalos, L O Wahlund, B Winblad, R C Petersen
}

J Neurol Neurosurg Psychiatry 2004;75:1275-1280. doi: 10.1136/jnnp.2003.015032

\begin{abstract}
Objective: The main aim of this collaborative study was to assess the comparability of the most commonly used criteria for mild cognitive impairment (MCI) by comparing the cognitive performance of patients with MCl from the Mayo Clinic (USA) and the Karolinska Institutet (Sweden). Methods: Standardised neuropsychological test scores were used to compare the two samples from the two institutions with regard to the number of cognitive domains in which performance was below 1.5 SD. Possible predictors for the conversion from $\mathrm{MCl}$ to Alzheimer's disease (AD) were assessed. Results: When the two institutions were considered together in the Cox proportional hazard model, the number of affected cognitive domains below 1.5 SD was a significant predictor of time to AD diagnosis with age, education, and $A P O E \& 4$ genotype entered into the same model as covariates. The number of affected cognitive areas remained as a significant predictor when the institutions were considered separately. The logistic regression model of conversion to $A D$ showed that only tests assessing learning and retention were predictors of developing $A D$.

Conclusions: Differences in population as well as in methodology of case ascertainment as well as other aspects may account for the observed variability between samples of patients with $\mathrm{MCl}$. The number of impaired cognitive factors at baseline can predict the progression from $M C l$ to $A D$. Furthermore, tests assessing learning and retention are the best predictors for progression to $A D$.
\end{abstract}

See end of article for authors' affiliations

Correspondence to: $\mathrm{O}$ Almkvist, Division of Clinical Geriatrics, Huddinge University Hospital B84, S-14186 Stockholm, Sweden; ove. almkvist@neurotec.ki.se

Received 2 May 2003 In revised form 28 November 2003 Accepted 1 December 2003

I recent years, the concept of mild cognitive impairment (MCI) has become increasingly popular. ${ }^{1}$ MCI refers to a transitional state between normal ageing and early Alzheimer's disease $(\mathrm{AD})$ whereby the subjects have intermediate cognitive impairments, usually involving memory but of insufficient magnitude to constitute the diagnosis of dementia or $\mathrm{AD}^{2}$ While there is good agreement on the concept of MCI, there is considerable variability concerning the specific diagnostic criteria and the rates of progression of subjects from MCI to $\mathrm{AD}^{3}{ }^{4}$

While most studies have documented an increased rate of progression in subjects with MCI, there is considerable variability in the literature..$^{5-7}$ Several possible explanations for this variability have been proposed including the sources of recruitment of the subjects and the specific diagnostic criteria employed. A recent international conference on MCI highlighted the heterogeneity of the term. ${ }^{1}$ In particular, there have been notable differences in the outcomes of subjects studied in the USA and Europe, and thus far, there has been no cross-national comparison of subjects with MCI .

This study aimed to address two of the major issues involved in the variability among the results of studies reported in the literature: subject population and diagnostic criteria. A group of subjects with MCI from the Mayo Clinic (MC) in Rochester, MN, USA, who have been studied for up to 15 years was compared with a group of subjects with MCI recruited by an academic medical centre, the Karolinska Institute (KI), in Stockholm, Sweden. The KI has a long history of studying subjects with intermediate stages of cognitive impairment and has extensive longitudinal data on the outcome of these subjects. The nature of the subject groups varies between these two institutions and served as a basis for investigating the difference in diagnostic criteria. This comparison allowed an evaluation of the role of source of recruitment of the subject population and varying of the criteria to assess the core features of the syndrome, as well as determination of the factors that play a significant role in predicting progression to $\mathrm{AD}$.

\section{PATIENTS AND METHODS}

Study sample

The two study groups consisted of 170 subjects with MCI from MC and 133 from KI. The demographic details of the subjects of both study groups as well as their APOE $\varepsilon 4$ genotypes are shown in table 1 .

\section{Mayo Clinic}

The MC subjects were recruited through the Mayo Clinic Alzheimer's Disease Center/Alzheimer's Disease Patient Registry (ADC/ADPR) using a standardised clinical proto$\mathrm{col}^{8}{ }^{9}$ A more detailed description of the recruitment procedure has been reported elsewhere. ${ }^{10}$ The commonest recruitment scheme involved screening of patients who were examined by primary care physicians for periodic general medical evaluation. On recruitment, patients were seen by a behavioural neurologist who obtained a medical history from the patients and corroborating sources, performed the Short Test of Mental Status, ${ }^{10}{ }^{11}$ Hachinski Ischemic Scale, ${ }^{12}$ and a neurological examination. Subjects were diagnosed with MCI if they met the following criteria:

- memory complaint, preferably corroborated by an informant

- objective memory impairment for age and education

- essentially normal general cognitive functions

- largely normal activities of daily living

- not demented.

At the MC, the diagnosis of MCI was based on the clinical judgement of a consensus committee comprised of behavioural neurologists, neuropsychologists, geriatricians,

Abbreviations: AD, Alzheimer's disease; AVLT, Auditory Verbal Learning Test; CDR, Clinical Dementia Rating (scale); KI, Karolinska Institute; MC, Mayo Clinic; MCFS, Mayo Cognitive Factor Scores; MCl, mild cognitive impairment; WAIS-R, Wechsler Adult Intelligence ScaleRevised; WMS-R, Wechsler Memory Scale-Revised 
Table 1 Demographic variables of the two study groups

\begin{tabular}{|c|c|c|c|}
\hline & $\begin{array}{l}M C \text { group } \\
(n=170)\end{array}$ & $\begin{array}{l}\mathrm{KI} \text { group } \\
(\mathrm{n}=133)\end{array}$ & $p$ value \\
\hline Age (years)* & $\begin{array}{l}78.5(8.4) ; \\
50-98\end{array}$ & $\begin{array}{l}69.5(5.8) ; \\
59-81\end{array}$ & $<0.0001$ \\
\hline Education (years)* & $\begin{array}{l}13.2(3.1) ; \\
4-20\end{array}$ & $\begin{array}{l}9.3(3.0) ; \\
5-18\end{array}$ & $<0.0001$ \\
\hline $\operatorname{Sex}(M / F)$ & $70 / 100$ & $74 / 59$ & $<0.06$ \\
\hline MMSE* & $\begin{array}{l}26.0(2.4) \\
18-30\end{array}$ & $\begin{array}{l}25.2(2.7) \\
17-30\end{array}$ & $<0.05$ \\
\hline Follow up (months) ${ }^{*}$ & $\begin{array}{l}37.50(17.72) ; \\
9-128\end{array}$ & $\begin{array}{l}27.55 \text { (17.72); } \\
8-84\end{array}$ & $<0.005$ \\
\hline \multicolumn{4}{|l|}{ APOE $\& 4$ genotype } \\
\hline$\varepsilon 4 / 4$ & $12(7.1 \%)$ & $9(6.8 \%)$ & \\
\hline$\varepsilon 4 / 3$ & $50(29.4 \%)$ & 35 (26.3\%) & \\
\hline$\varepsilon 2 / 4$ & $7(4.1 \%)$ & $4(3 \%)$ & NS \\
\hline$\varepsilon 3 / 3$ & 60 (35.4\%) & 41 (30.8\%) & \\
\hline$\varepsilon 2 / 3$ & $11(6.5 \%)$ & $11(8.3 \%)$ & \\
\hline Missing & $30(17.5 \%)$ & $33(24.8 \%)$ & \\
\hline
\end{tabular}

${ }^{*}$ Mean (SD); range.

KI, Karolinska Institutet; MC, Mayo Clinic; MMSE, Mini Mental State Examination; NS, not significant.

psychiatrists, and nurses. There were no psychometric cut-off scores for the diagnosis; rather, the clinicians determined if the subject met the criteria outlined above. At each evaluation, the neuropsychological tests were administered by experienced psychometrists who were supervised by two clinical neuropsychologists. Patients were seen for follow up on an approximately annual basis.

\section{Karolinska Institutet}

The 133 patients with MCI were referred from the primary care centres in the community to the Geriatric Clinic, Huddinge University Hospital for investigation of suspected dementia. The objective impairment of the patients was 1.5 SD below the average for their age on neuropsychological tests, representing one or more domains of cognition as described by Wahlund et al. ${ }^{13}$ All subjects were examined according to the same comprehensive procedure, ${ }^{13}$ which included a physical examination, evaluation of neurological status, psychiatric status, review of previous case records, blood test, urine analysis, cerebrospinal fluid analysis, routine electrocardiogram, routine electroencephalogram, magnetic resonance imaging, single photon emission computed tomography, and the Mini Mental State Examination (MMSE). ${ }^{14}$ The neuropsychological examination was performed by experienced neuropsychologists. All subjects lived independently in the community and in all cases a close informant was interviewed to gather information about the functional status of the patient.

\section{Diagnostic procedure}

The diagnostic criteria for MCI at both institutions are shown in table 2. Follow up diagnoses of dementia and $\mathrm{AD}$ were made according to the DSM-III-R, ${ }^{15}$ for dementia and the NINCDS-ADRDA ${ }^{16}$ for $\mathrm{AD}$ at both institutions. For assessment of interference with activities of daily living (ADL), the Clinical Dementia Rating (CDR) scale was used at the MC and clinical judgement at KI.

\section{Follow up procedure}

Subjects were re-evaluated every 12-18 months at the MC and received an abbreviated clinical evaluation at that visit as described previously. ${ }^{10}$ The initial and the follow up examinations included the same psychometric routine as well as an informant interview regarding behavioural and functional status. In these cases, a consensus diagnosis was again rendered as described above. At the KI, patients with MCI were re-evaluated every twelve months. The evaluation
Table 2 Diagnostic criteria for mild cognitive impairment at the Mayo Clinic and the Karolinska Institutet

\begin{tabular}{ll}
\hline Mayo Clinic & Karolinska Institutet \\
\hline $\begin{array}{l}\text { Complaints about memory } \\
\text { Activities of daily living }\end{array}$ & $\begin{array}{l}\text { Complaints about memory } \\
\text { No social interference with } \\
\text { daily life as reported }\end{array}$ \\
$\begin{array}{l}\text { General cognitive function } \\
\text { normal }\end{array}$ & $\begin{array}{l}\text { Decline in any cognitive domain } \\
\text { (1.5 SD below age matched controls) } \\
\text { Nomory for age abnormal }\end{array}$ \\
\hline
\end{tabular}

included the same comprehensive clinical procedure applied at baseline as well as neuropsychological assessment. None of the patients from the two institutions was on acetylcholinesterase inhibitor treatment at initial evaluation.

\section{Neuropsychological assessment}

Neuropsychological assessment at both centres comprised a common set of measures, which facilitated the crosscomparison. The set included the Wechsler Adult Intelligence Scale-Revised (WAIS-R) ${ }^{17}$ (information, digit span, similarities, block design and digit symbol, and digit span subtests), immediate and delayed recall of the story from the Wechsler Memory Scale-Revised (WMS-R) ${ }^{18}$ and Auditory Verbal Learning Test (AVLT). ${ }^{19}$ We excluded tests that were not carried out in a similar way at both institutions.

\section{Standardisation of the samples Mayo Clinic}

Mayo's Older Americans Normative Studies (MOANS) standard scores were calculated for the MC patients. MOANS scores provide norms for a number of cognitive tests (core battery) that are commonly used to evaluate individuals from age 55 to $97 .^{2021}$ Smith et $a^{22-24}$ have previously demonstrated that five cognitive factors underlie the MOANS core battery in both a normative and a clinical sample. These factors, labelled the five Mayo Cognitive Factor Scores (MCFS), include verbal comprehension, perceptual organisation, attention/concentration, learning, and retention, as described elsewhere. ${ }^{2}$ The tests included in each factor are further detailed in table 3.

\section{Karolinska Institutet sample}

The KI MCI neuropsychological scores were standardised by using a control group of subjects consisting of the patients' relatives, members of the Swedish Pensioner Society in the Huddinge community, and non-mutation carriers from $\mathrm{AD}$ families.

For comparisons between the MC and KI groups, we organised the neuropsychological measures according to the MCFS. We assumed that scores below 1.5 SD according to age and years of education was a sign of cognitive abnormality. We were thus able to calculate "the number of impaired cognitive domains", with scores ranging from 0 to 5. For the purpose of comparison, we restricted our analysis to those patients who had 1, 2, or 3 cognitive domains affected. These patients represented the major proportion overall in the cognitive spectrum.

\section{Statistical procedure}

Student's $t$ test was used to analyse the differences between the groups with regard to the demographic and standardised $\mathrm{z}$ scores neuropsychological variables. APOE $\varepsilon 4$ genotype distributions were compared with $\chi^{2}$ test (see table 1). A Kaplan-Meier survival function with dementia diagnosis 
Table 3 Neuropsychological standardised z scores of the two study groups with mild cognitive impairment

\begin{tabular}{|c|c|c|c|}
\hline Cognitive function & Tests & $\begin{array}{l}\text { Mayo Clinic } \\
(n=170)\end{array}$ & $\begin{array}{l}\text { Karolinska Institutet } \\
(n=133)\end{array}$ \\
\hline Verbal comprehension & $\begin{array}{l}\text { WAIS-R: information } \\
\text { WAIS-R: similarities }\end{array}$ & $\begin{array}{l}-0.36(2.66) \\
-0.01(0.97)\end{array}$ & $\begin{array}{l}-1.45(1.04)^{*} \\
-2.15(0.98)\end{array}$ \\
\hline Attention & $\begin{array}{l}\text { WAIS-R: digit symbol } \\
\text { WAIS-R d diait }\end{array}$ & $\begin{array}{l}-0.04(0.98) \\
-0.08(233)\end{array}$ & $\begin{array}{l}-1.13(0.96)^{*} \\
-4.01(1.98)^{* *}\end{array}$ \\
\hline \multirow{3}{*}{$\begin{array}{l}\text { Perceptual organisation } \\
\text { Learning and } \\
\text { memory }\end{array}$} & $\begin{array}{l}\text { WAIS-R: digit span } \\
\text { WAlock design }\end{array}$ & $-0.21(1.03)$ & $-0.24(0.90)^{*}$ \\
\hline & $\begin{array}{l}\text { WMS-R: logical memory } \\
\text { (immediate story recall) }\end{array}$ & $-0.39(2.00)$ & $-6.45(1.25)^{\star *}$ \\
\hline & AVLT: learning over trials & $-1.56(1.65)$ & $-1.25(1.35)^{*}$ \\
\hline \multirow[t]{2}{*}{ Retention } & $\begin{array}{l}\text { WMS-R: logical memory } \\
\text { (delayed story recall) }\end{array}$ & $-1.90(2.70)$ & $-2.3(1.7)^{\star *}$ \\
\hline & AVLT: delayed recall & $-1.03(1.52)$ & $-2.3(1.9)^{* *}$ \\
\hline \multicolumn{4}{|c|}{$\begin{array}{l}\text { All values are mean (SD). } \\
{ }^{*} \mathrm{p}<0.05 \text {. } \\
\text { ** } \mathrm{p}<0.01 \text {. } \\
\text { AVLT, Auditory Verbal Learning Test; WAIS-R, Wechsler Adult Intelligence Scale-Revised; WMS-R, Wechsler } \\
\text { Memory Scale-Revised. }\end{array}$} \\
\hline
\end{tabular}

used as "an event" was calculated. A global test for proportions was used to check the assumptions of the Cox proportional hazard model, which was then used to assess risk factors for incident $\mathrm{AD}$ among the patients with MCI. Potential risk factors in the hazards analysis included age, years of formal education, and the APOE \&4 genotype. The choice of these covariates was based on their associations with the outcome of $\mathrm{AD}$ in the literature. ${ }^{25}$ We also examined the $\mathrm{AD}$ diagnosis as a discrete binary outcome. Logistic regression analyses were conducted with the same covariates after restricting the group with MCI to patients who had completed 50 months follow up or developed $\mathrm{AD}$ before that time.

\section{RESULTS}

\section{Baseline clinical features}

There were differences between the KI and MC groups in terms of age, education, and MMSE as shown in table 1 . The $A P O E$ \&4 genotype was evaluated in a total of 240 patients from MC and KI ( see table 1). The distribution of the APOE \&4 genotype frequencies for the two institutions was not different $\left(\chi^{2}=1.66 ; \mathrm{df}=3 ; \mathrm{p}>0.05\right)$. On the other hand, there was no association between the $\varepsilon 4$ allele and the outcome of $\mathrm{AD}$.

\section{Prediction of AD}

Figure lA shows the survival function when all the patients from the two institutions are considered together, without taking into account "the number of impaired cognitive functions" at baseline. The two curves differed significantly $(\log$ rank $=11.8 ; \mathrm{df}=1 ; \mathrm{p}<0.0001)$. Since the major proportion of patients had two impaired cognitive domains, we calculated Kaplan-Meier survival curves for patients from MC and KI (fig 1B). The difference between the two curves was significant $(\log$ rank $=3.61 ; \mathrm{df}=2 ; \mathrm{p}<0.05)$. When the two institutions were considered together in a Cox proportional hazard model, "the number of affected cognitive factors" was a significant predictor of time to AD diagnosis with age (not significant (NS)), number years of formal education (NS), and $A P O E$ \&4 genotype (NS) entered into the same model as covariates. The only significant covariate in the model was "institution" (MC $v$ KI). Additional Cox analyses were conducted for the two institutions separately. When the model was adjusted for each institution independently for age, years of formal education, and APOE $\varepsilon 4$ genotype, the number of impaired cognitive domains remained a significant predictor. In addition, the APOE $\varepsilon 4$ genotype was a significant predictor for the KI group when investigated in isolation.

For the two groups followed up at two years, the number of impaired cognitive factors $(-1.5 \mathrm{SD})$ at baseline led to sensitivity of $80 \%$ and specificity of $75 \%$ for the diagnosis of $\mathrm{AD}$, including a progressive increase in sensitivity and a decrease in specificity with two or three "cognitive factors below $1.5 \mathrm{SD}^{\prime \prime}$.
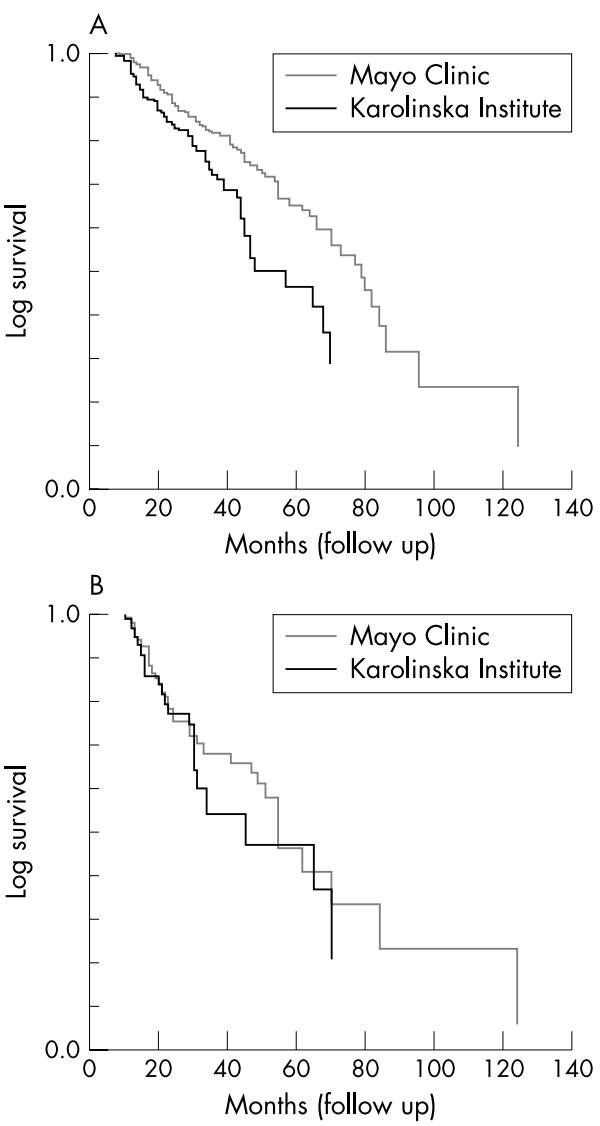

Figure 1 (A) Kaplan-Meier survival curve of probability of developing Alzheimer's disease (AD) over 140 months (Mayo Clinic and Karolinska Institutet patients with mild cognitive impairment (MCI) combined). (B) Kaplan-Meier survival curves of probablility of developing $A D$ over 50 months (Karolinska Institutet) and over 140 months (Mayo Clinic) in subjects with $\mathrm{MCl}$ with two cognitive functions below $1.5 \mathrm{SD}$ at baseline. 
Neuropsychological measures were also entered into a logistic model of AD outcome (table 4). The results revealed that WMS: delayed recall was the only significant predictor of $\mathrm{AD}$, when the two samples were added together. When the KI MCI sample was considered in isolation, the predictive values were AVLT: delayed recall, WMS: delayed recall, WAIS-R: information, and WAIS-R: digit span. Similarly, when the MC sample was analysed independently, the best two predictors were WMS: delayed recall and WMS: immediate recall. Neither age or education nor $A P O E \& 4$ genotype was significant in any of the three models.

\section{DISCUSSION}

This study addressed two issues concerning the variability of results in the literature concerning MCI: (a) the sources of subject recruitment, and $(b)$ the diagnostic criteria.

The MC recruited its patients from a primary care setting by proactively reviewing medical records to detect any suspicion of the subjects having a cognitive concern-if there was a suggestion that the subject might be impaired, permission was sought to approach the individual for participation in the research study. This design allowed for detecting cognitive impairment prior to the point at which subjects would be referred either by themselves, their family, or physicians. The KI is an academic medical centre where subjects were recruited from consecutive clinical patients referred to the geriatric department by primary care physicians. Therefore, it is possible that these subjects may have had more advanced cognitive symptoms. When common sets of neuropsychological measures were used to compare the samples, it became apparent that the KI subjects with MCI were slightly more impaired than the MC subjects. The indices of general cognition and individual neuropsychological test performance revealed more impairment in the KI subjects, yet the experienced clinicians in this institute did not feel that the subjects were demented. These data document that individual subjects may have various types of MCI and their clinical profiles may vary as a function of the recruitment strategy used to enrol them in the study. Slightly different populations of subjects can have a significant impact on the clinical profile of the subjects recruited.

In addition to the differences in the recruitment procedures, the subjects were ascertained with slightly different criteria. The MC subjects with MCI were impaired primarily in the memory domain (amnestic MCI), while other cognitive domains were relatively intact. ${ }^{1}$ The other non-memory cognitive domains were not statistically normal, but the impairments in these other areas were not believed to be clinically significant. However, the KI subjects were impaired slightly in multiple cognitive domains but these were not of sufficient magnitude to constitute the diagnosis of dementia.
Consequently, these subjects would be more characteristic of the subjects with multiple domain MCI described previously. ${ }^{1}$ This type of variability has been seen in other studies. ${ }^{72}$ In addition, the assessment of interference with daily living was different, at the MC the CDR scale was used and at KI clinical judgement was used. The latter procedure may be more conservative in terms of degree of change required than a procedure that make use of a specified scale such as the CDR scale. This might have also resulted in the sample of patients at KI being slightly more impaired compared with those from MC.

Using the number of impaired cognitive domains at baseline, the Kaplan-Meier curves demonstrated that it was possible to predict the progression from MCI to AD in both groups. The survival curves for the two institutions were slightly different indicating that conversion from MCI to AD depended on the particular institution, and that the severity of impairment in the various domains was also different between the two institutions.

It is interesting to note that clinical heterogeneity was also observed when the two institutions were considered separately. While some controversy exists as to the precise characterisation of subjects with MCI, these data suggest that detailed neuropsychological testing can accurately identify individuals experiencing mild or even unrecognised cognitive impairment in the primary care setting who are at a greater risk of developing AD. ${ }^{27-29}$ However, excessive reliance on neuropsychological data in the absence of the judgement of clinicians can lead to exaggerated inclusion of patients into the MCI cohort. ${ }^{5}$ It is important to note that the diagnosis of MCI was made on a clinical basis at both institutions. While the neuropsychological tests were supportive of the clinician's judgement, the final diagnosis of MCI was rendered by clinicians at both institutions. This likely led to the stability of the diagnosis and the reliability of the progression of the subjects over time.

To highlight this point, a recent study by Ritchie et al ${ }^{6}$ documented the finding that when neuropsychological criteria are applied retrospectively, this type of MCI diagnosis can be unreliable. In this study, the authors set the memory criteria at 1 SD below the mean and required other nonmemory cognitive domains to be at the mean or above. In so doing, they demonstrated that subjects who fit this profile were uncommon and generally did not progress to dementia. Petersen et al showed that when clinical judgement was invoked in characterising patients with a primary memory impairment and only slight impairments in other cognitive domains, subjects progressed at a regular rate to $\mathrm{AD}$. These individuals did not have statistically normal performance in other non-memory domains; however, the clinicians did not feel these other impairments were of sufficient magnitude to constitute dementia. The present study corroborated these

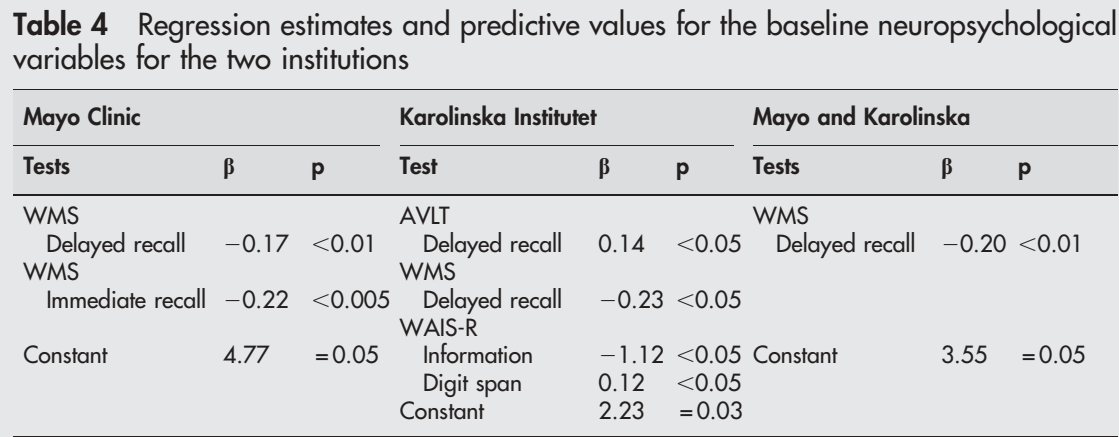


findings by using clinical judgement to augment the neuropsychological data.

There were also a few other methodological differences between the two institutions. For example, the use of five MCFS allowed the MC investigators to assess quantitatively the MC cognitive profile, whereas the KI neuropsychological assessment battery was based on clinical evaluation of abnormal test results depending upon the age and education of the subject.

The regression model demonstrated that tests assessing learning and retention were the best predictors for progression to $\mathrm{AD}$ when the two samples were considered together. This finding has been reported in previous research. ${ }^{28}{ }^{30-32}$ In fact, Bozoki et $a l^{32}$ and Albert et $a l^{33}$ have demonstrated that when other cognitive functions beyond memory become significantly impaired, the likelihood of more rapid progression to dementia or $\mathrm{AD}$ increases.

The finding that $A P O E \varepsilon 4$ was a significant predictor in the KI but not in the MC group may reflect the influence of the $\varepsilon 4$ allele in the early stages of the disease since the KI patients were younger than the MC subjects. Other work has indicated that the $A P O E \& 4$ allele is not only a risk factor for developing $\mathrm{AD}$ but may also influence the age of expression. ${ }^{34}$ Therefore, the age differences in these two samples might have been significant.

In summary, this study demonstrated that MCI populations can be compared cross-nationally. The two research groups are experienced in evaluating cognitive impairment and dementia and characterised two groups of subjects who were felt to be impaired but did not fulfil the criteria for dementia. One research group used a memory predominant set of criteria (amnestic MCI) and their results demonstrated a regular progression to dementia. The other research group used a definition of MCI which included mild impairments in multiple cognitive domains (multiple domain MCI). ${ }^{4}$ While both sets of criteria are valid, they led to slightly different populations of subjects. In addition, the degree of impairment of the two samples also had an impact on the characterisation of the two groups and their progression to dementia. The results of the present study lend support to the idea that although the MCI concept covers a heterogeneous group of patients, it still has predictive value for future development. However, at the same time it is an unanswered question whether subgroups of MCI could be separated as suggested recently by an international working group ${ }^{1}$ and whether these subgroups differ with regard to future outcome.

To conclude, our study indicates that MCI is a viable concept in different clinical settings and also emphasises the importance of recognising the various factors that can have an impact on the characterisation of the clinical groups being studied and on their outcome. As greater attention is paid to these sources of variability, the concept of MCI can be refined and the appropriate selection of subjects for clinical trials enhanced.

\section{ACKNOWLEDGEMENTS}

We express our gratitude to all the patients for their contribution.

\footnotetext{
Authors' affiliations

E Arnáiz, O Almkvist, L O Wahlund, B Winblad, Karolinska Institutet, Neurotec Department, Division of Clinical Geriatrics, Huddinge University Hospital, Stockholm, Sweden

R J Ivnik, Department of Psychiatry and Psychology, Mayo Clinic and Mayo Foundation, Rochester, MN, USA

E G Tangalos, Department of Internal Medicine, Mayo Clinic and Mayo Foundation, Rochester, MN, USA
}

R C Petersen, Department of Neurology, Mayo Clinic and Mayo Foundation, Rochester, MN, USA

This work was supported by the Margit and Folke Pehrzon Foundation, the Gamla Tiännarinor Foundation, Swedish Research Council, and Alzheimerfonden.

The Mayo work was supported by grants from the National Institute on Aging, Mayo Clinic Alzheimer's Disease Center AG 16574 and Alzheimer's Disease Patient Registry AG 06786.

Competing interests: Ronald $C$ Petersen receives funding for research on mild cognitive impairment through a consortium involving the National Institute on Aging and the University of California-San Diego from Pfizer, Inc and Eisai, Inc. He also receives consulting fees from Elan Pharmaceuticals/Wyeth, Inc. He has received honoraria for speaking from several pharmaceutical companies including Pfizer, Eisai, Novartis and Janssen, but these are through national organisations such as the American Academy of Neurology or through universities and medical centres using unrestricted educational grants. He has attended a symposium on mild cognitive impairment sponsored by Mount Sinai Medical Centre in Miami which was supported by pharmaceutical company grants. He is a consultant to the Loma Linda University.

\section{REFERENCES}

1 Petersen RC, Doody R, Kurz A, et al. Current concepts in mild cognitive impairment. Arch Neurol 2001;58:1985-992.

2 Smith GE, Petersen RC, Parisi JE, et al. Definition, course, and outcome of mild cognitive impairment. Aging Neuropsychol Cogn 1996;3:141-7.

3 Dawe B, Procter A, Philpot $M$, et al. Concepts of mild memory impairment in the elderly and their relationship to dementia: a review. Int J Geriatr Psychiatry 1992:7:473-9.

4 Petersen RC. Conceptual overview. In: Petersen RC, ed. Mild Cognitive Impairment: Aging to Alzheimer's Disease. New York: Oxford University Press, 2003.

5 Petersen RC, Morris JC. Clinical features. In: Petersen RC, ed. Mild Cognitive Impairment: Aging to Alzheimer's Disease. New York: Oxford University Press, 2003.

6 Ritchie K, Touchon J. Mild cognitive impairment: conceptual basis and current nosological status. Lancet 2000;355:225-8.

7 Petersen RC, Smith GE, Ivnik RJ, et al. Memory function in very early Alzheimer's disease. Neurology 1994;44:867-72.

8 Petersen RC, Smith GE, Ivnik, et al. Apolipoprotein E status as a predictor of the development of Alzheimer's disease in memory-impaired individuals. JAMA 1995;273:1274-8

9 Petersen RC, Smith GE, Warring SG, et al. Mild cognitive impairment: clinical characterisation and outcome. Arch Neurol 1999;56:303-8.

10 Kokmen E, Naessens JM, Offord KP. A Short test of mental status: description and preliminary results. Mayo Clin Proc 1987;62:281-8.

11 Kokmen E, Smith GE, Petersen RC, et al. The short test of mental status: correlations with standardized psychometric testing. Arch Neurol 1991;48:725-8.

12 Rosen W, Terry R. Pathological verification of ischemic scores differentiation of dementias. Ann Neurol 1980;7:486-8.

13 Wahlund LO, Pihlstrand E, Eriksdotter Jönhagen M. Mild cognitive impairment: experience from a memory clinic. Acta Neurol Scand 2003; 107(suppl 179):21-4.

14 Folstein MF, Folstein SE, McHugh PR, et al. Mini-Mental State: a practical method for grading the cognitive state of patients for the clinician. J Psychiatr Res 1975;12:189-98.

15 American Psychiatric Association. Diagnostic and Statistical Manual of Mental Disorders, 4th ed. Washington, DC: American Psychiatric Association, 1994.

16 McKhann G, Drachman D, Folstein M, et al. Clinical diagnosis of Alzheimer's Disease: report of the NINCDS-ADRDA work group under the auspices of Department of Health and Human Services Task Forces on Alzheimer's Disease. Neurology 1984;34:939-44.

17 Wechsler D. Wechsler Adult Intelligence Scale-Revised Manual. San Antonio, TX: Psychologcial Corp, 1981.

18 Wechsler D, Stone CP. Wechsler Memory Scale Manual. New York, NY: Psychological Corp, 1973.

19 Rey A. L'examen clinique en psychologie. Paris: Presses Universitaries de France, 1959.

20 Ivnik RJ, Malec JF, Smith GE, et al. Mayo's Older Americans Normative Studies: WAIS-R norms for ages 56 to 97 . Clin Neuropsychol 1992;6(suppl): 1-30.

21 Ivnik RJ, Smith GE, Lucas JA, et al. Free and cued selective reminding test: MOANS norms. J Clin Exp Neuropsychol 1997;19:676-91.

22 Smith GE, Ivnik RJ, Malec JF, et al. Mayo's Older Americans Normative Studies (MOANS): Factor structure of a core battery. Psychol Assess 1992;4:382-90.

23 Smith GE, Ivnik RJ, Malec JF, et al. Factor structure of the MOANS core battery; replication in a clinical sample. Psychol Assess 1993:5:121-4.

24 Smith GE, lvnik RJ, Malec JF, et al. Mayo Cognitive Factor Scales: Derivation a short battery and norms for factors scores. Neuropsychology 1994;8: 194-202 
25 Tierney MC, Szalai JP, Snow, et al. Prediction of probable Alzheimer's Disease in memory-impaired patients: a prospective longitudinal studies. Neurology 1996;46:661-5.

26 Almkvist O, Basun H, Bäckman L, et al. Mild cognitive impairment-an early stage of Alzheimer's disease? J Neural Transm 1998:54:21-9.

27 Rubin EH, Morris JC, Grant EA, et al. Very mild senile dementia of Alzheimer type. Clinical assessment. Arch Neurol 1989;46:379-86.

28 Jacobs DM, Sano M, Dooneief G, et al. Neuropsychological detection and characterization of preclinical Alzheimer's disease. Neurology 1995:45:957-62.

29 Elias MF, Beiser A, Wolf PhA. The pre-clinical phase of Alzheimer's disease Arch Neurol 2000;57:808-13.
30 Arnáiz E, Blomberg M, Fernaeus SE, et al. Psychometric discrimination of Alzheimer's disease and mild cognitive impairment. Alzheimer's Reports 2000;3:97-104.

31 Grober E, Lipton RB, Hall C, et al. Memory impairment on free and cued selective reminding predicts dementia. Neurology 2000;54:827-32.

32 Bozoki A, Giordani B, Judith L, et al. Mild cognitive impairments predicts dementia in nondemented elderly patients with memory loss. Arch Neurol 2001;58:411-16.

33 Albert MS, Moss MB, Tanzi R, Jones K. Preclinical prediction of AD using neuropsychological tests. J Int Neuropsychol Soc 2001;7:631-9.

34 Lambert JC, Araria-Goumidi L, Myllykangas L, et al. Contribution of APOE promoter polymorphisms to Alzheimer's disease risk. Neurology 2002;59:59-66.

\section{$\mathrm{ECHO}$}

\section{Sydenham's chorea may be relevant to common childhood idiopathic conditions}

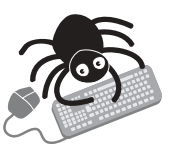

Please visit the Journal of Neurology, Neurosurgery, and Psychiatry website [www. innp.com] for a ink to the full text of this article.
D octors studying neurological disorders arising in children after streptococcal infections have suggested that understanding how these are expressed could greatly improve our knowledge of common but unexplained childhood movement and psychiatric conditions.

The disorders in question are dyskinesias which routinely occur with disabling psychiatric conditions - the legacy of infection with $\beta$ haemolytic streptococci. Doctors at a tertiary referral centre found a wide range of psychiatric conditions among 40 children seen between 1999 and 2002 with neurological complications after such infection. Sex differences and a genetic component were also evident.

Chorea-including Sydenham's chorea, the classic dyskinesia after streptococcal infection (20 patients) - and motor tics (16,40\%) were the most common neurological complications. Chorea occurred mostly in girls (65\%) and tics in boys (69\%). Only children with chorea had systemic complications of infection-carditis and arthritis-which always preceded neurological complications. Acute emotional or behavioural changes became evident in 33 $(83 \%)$ children after their streptococcal infection, with emotional lability, anxiety, obsessive compulsive disorder, and depression occurring most commonly.

All 40 children were positive for $\beta$ haemolytic streptococcal antibody, but $34(85 \%)$ had clinical evidence of such infection before the neurological disorder appeared, after a mean interval of 18.9 (range 1-67) days. Almost three quarters have continuing symptoms of movement disorders after an average of two and a half years, some for as long as 13 years. Forty per cent had a family history of psychiatric or movement disorders in first degree relatives and a similar proportion autoimmune complications in first or second degree relatives.

A Dale RC, et al. Archives of Disease in Childhood 2004;89:604-610. 